\title{
Half-Round Inductive Obstacles in Rectangular Waveguide
}

\author{
D. M. Kerns
}

(January 30, 1960)

\begin{abstract}
Formulas are derived for the accurate calculation of the Jowest-mode, lumped-element representation of perfectly conducting half-round inductive obstacles in rectangular waveguide. These obstacles consist of either one or two opposed semicircular cylindrical indentations extending across the narrow sides of the waveguide. They seem especially suitable for use as precise calculable standards of reflection or impedance in waveguide. Schwinger's integral equation approach $[1,2]^{1}$ is used to obtain stationary expressions for the desired parameters as functionals of the surface currents on the obstacles. Upper bounds are obtained for one of the two parameters. Explicit formulas are derived for the values of the parameters under the assumption of $n$-term Fourier sine-series expansions for the obstacle currents. Rapid convergence is indicated by numerical evaluations for $n=1,2$, and 3 . In the process of obtaining expressions suitable for numerical calculation, an expansion (believed to be new) of the Green's furction of the problem is obtained and the sums of certain infinite series of Bessel's functions occurring in this expansion are expressed in terms of definite integrals. A brief numerical table of these sums, sufficient for the evaluation of the $n=1$ approximation, is included.
\end{abstract}

\section{Introduction}

In this paper formulas are derived for the accurate calculation of lowest-mode, lumpedelement parameters for what may be identified as "single half-round" and "double half-round" inductive obstacles in rectangular waveguide. As shown in figure 1, the obstacles consist of semicircular cylindrical indentations extending across the narrow sides of the waveguides.

The particular geometry considered seems especially suitable for obstacles to be used as calculable standards of reflection or impedance in waveguide. The geometry is well suited to electroforming, so that obstacles may be fabricated by this process as well as by machining. For obstacles producing standing-wave ratios of moderate values, the obstacle radius is large compared to high-standard machining tolerances, so that unduly close tolerances are not required. A change in waveguide cross section before and after the obstacle is avoided, eliminating the need for waveguide components in odd sizes and permitting maximum flexibility of interconnection.

In the present work the obstacle and waveguide surfaces are assumed perfectly conducting.

The Waveguide Handbook [3] contains approximate formulas for semi-elliptical obstacles, ${ }^{2}$ which can be specialized to apply to the present problem. However, for the contemplated use in standards work, formulas with very much greater accuracy $\left(10^{3}\right.$ or $10^{4}$ times greater $)$ are wanted.

Brief descriptions of the present and some related work have been given previously [4].

\section{Formulation of Problem}

We employ the customary complex electric and magnetic field vectors $\boldsymbol{E}=\boldsymbol{E}(\boldsymbol{r}), \boldsymbol{H}=\boldsymbol{H}(\boldsymbol{r})$, which satisfy Maxwell's equations in the form

$$
\left.\begin{array}{r}
\nabla \times \boldsymbol{E}=-j \omega \mu \boldsymbol{H}, \\
\nabla \times \boldsymbol{H}=j \omega \epsilon \boldsymbol{E}+\boldsymbol{J},
\end{array}\right\}
$$

\footnotetext{
1 Figures in brackets indicate the literature references at the end of this paper.

2 The pertinent formulas are afflicted with a number of misprints; correct forms of these results for half-round obstacles are given later in this paper.
} 
under the assumption of time-dependence represented by the (omitted) factor exp $(j \omega t)$. Here $t$ is the time, $\omega /(2 \pi)$ is the frequency, $j$ is the imaginary unit, $\boldsymbol{J}$ is the complex current-density vector, and $\mu$ and $\epsilon$ are respectively the permeability and the permittivity of the homogeneous, isotropic, nondissipative medium in the waveguide. The MKS system of units is employed.

As shown in figure 1, we choose a rectangular coordinate system $O x y z$ such that the interior of the waveguide is the space $0<x<a, \quad 0<y<b, \quad-\infty<z<\infty$ and the surfaces of the obstacles are given by the loci

$$
\left.\begin{array}{l}
R^{2}=x^{2}+z^{2}, \quad 0 \overline{<} x \overline{<} R, \\
R^{2}=(a-x)^{2}+z^{2}, \quad 0 \overline{<} a-x \overline{<} R
\end{array}\right\}
$$

in the double half-round case and by

$$
R^{2}=x^{2}+z^{2}, \quad 0 \overline{<} x \overline{<}
$$

in the single half-round case.
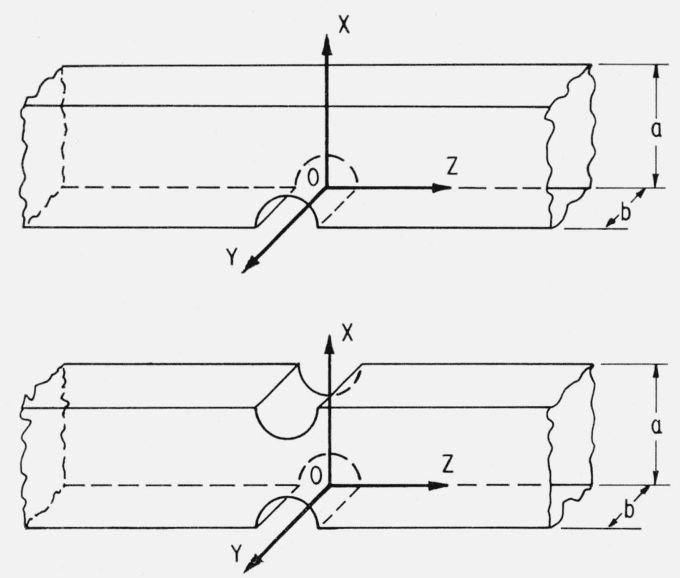

FIGURE 1. Single and double half-round inductive obstacles in rectangular waveguide.

The fields incident on the obstacles are, by hypothesis, to be in the $\mathrm{TE}_{10}$ mode. Travelingwave forms for this mode may be written

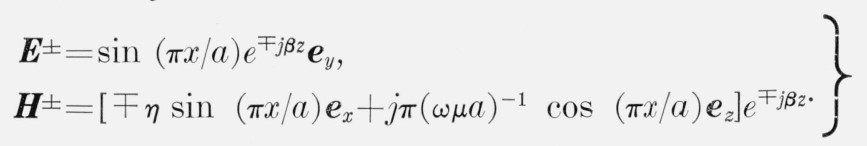

The upper and lower signs refer respectively to waves going in the positive and the negative $z$-directions; the phase constant $\beta$ is equal to $\left[k^{2}-(\pi / a)^{2}\right]^{1 / 2}$, where $k=\omega(\mu \epsilon)^{1 / 2}$; the wave-admittance $\eta=\beta /(\omega \mu)$; and $\boldsymbol{e}_{x}, \boldsymbol{e}_{y}, \boldsymbol{e}_{z}$ are the unit vectors of the system $O x y z$. It is assumed that $\pi<k a<2 \pi$, so that the $\mathrm{TE}_{10}$ mode, but no higher mode of the type $\mathrm{TE}_{n 0}$, will have real propagation.

We define "voltage" and "current" $v_{m}\left(z_{m}\right), i_{m}\left(z_{m}\right)$ for the $\mathrm{TE}_{10}$ mode by means of the equations

$$
\left.\begin{array}{l}
E_{m y}=v_{m}\left(z_{m}\right) \sin (\pi x / a) \\
H_{m x}=(-)^{m} i_{m}\left(z_{m}\right) \eta \sin (\pi x / a)
\end{array}\right\} m=1,2
$$

Here the indices $m=1,2$ refer respectively to the "left-hand" side $\left(z_{1}<-R\right)$ and the "righthand" $\left(z_{2}>R\right)$ of the obstacle considered, and $E_{m y}, H_{m x}$ are components (identified by the subscripts) of the $\mathrm{TE}_{10}$-mode part of whatever total electromagnetic field may be present in the waveguide at $z=z_{m}$. From (2.6) and (2.4) it follows that the waveguide characteristic impedance, defined as the value of $v_{m}\left(z_{m}\right) / i_{m}\left(z_{m}\right)$ for a pure traveling wave incident on the side $m$ of the obstacle, is equal to unity for $m=1,2$. 
The desired lowest-mode, lumped-element description of an obstacle is contained in its impedance matrix $Z$, which characterizes the linear relations

$$
v_{1}=Z_{11} i_{1}+Z_{12} i_{2}, \quad v_{2}=Z_{21} i_{1}+Z_{22} i_{2}
$$

imposed by the obstacle on the terminal variables $v_{m}, i_{m}$. The values of the $Z_{m n}$ depend upon the location of the terminal planes $z=z_{1}$ and $z=z_{2}$; in the present problem it turns out to be convenient to extrapolate these planes to $z_{1}=z_{2}=0$.

Now, the equality $Z_{11}=Z_{22}$ follows from the structural symmetry (and the symmetrical disposition of the terminal planes) with respect to the plane $z=0 ; Z_{12}=Z_{21}$ is assured by both symmetry and reciprocity; and, since losslessness has been assumed, $Z$ is pure imaginary. Thus $Z$ is of the form

$$
\left(\begin{array}{ll}
j X_{11} & j X_{12}, \\
j X_{12} & j X_{11}
\end{array}\right),
$$

and there are only two independent parameters to be determined.

We do not obtain formulas for $X_{11}$ and $X_{12}$ directly; but rather, to exploit the symmetry of the problem more fully, we consider the impedances obtaining under modes of excitation in which the electromagnetic field is either symmetric or antisymmetric with respect to the plane $z=0$. Thus in the symmetric case, $v_{1}(0)=v_{2}(0), i_{1}(0)=i_{2}(0)$, and the impedance may be written

$$
Z_{e e}=v_{1, e}(0) / i_{1, e}(0),
$$

where we have added a subscript distinguishing the "even" case. In the antisymmetric ("odd") case, $v_{1}(0)=-v_{2}(0), i_{1}(0)=-i_{2}(0)$, and the impedance may be written

$$
Z_{00}=v_{1,0}(0) / i_{1,0}(0) \text {, }
$$

again adding a distinguishing subscript. The relations

$$
2 j X_{11}=Z_{e e}+Z_{00}, \quad 2 j X_{12}=Z_{e e}-Z_{00}
$$

follow directly from (2.7), (2.8), and (2.9). (It may be noted that the symmetric and antisymmetric field distributions correspond to the eigenvectors of the matrix (2.8) and the quantities $Z_{e e}$ and $Z_{00}$ are the eigenvalues of this matrix.)

An equivalent network for the obstacles is of interest and is presented here for convenient reference. For a $T$-network, using the sign conventions shown in figure 2, we find

$$
Z_{1}=Z_{00}, \quad 2 Z_{2}=Z_{e e}-Z_{00},
$$

where $Z_{1}$ and $Z_{2}$ are respectively the series and the shunt elements of the symmetrical $T$. It may be remarked that the obstacles considered are called "inductive" because for small radii the important element, $Z_{2}$, is a positive reactance.

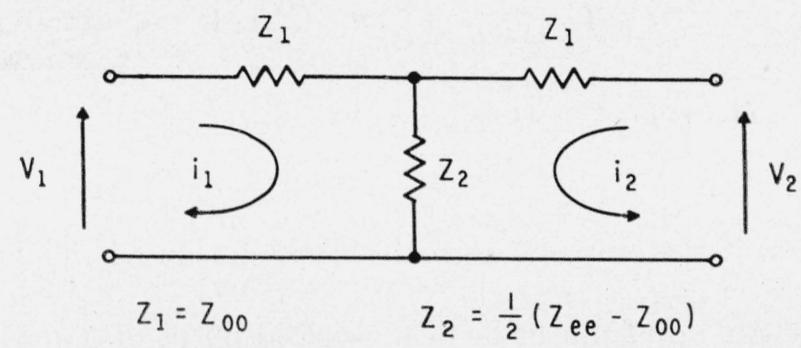

Figure 2. Equivalent network. 


\section{Analysis}

\subsection{Reduction to Scalar Form}

Maxwell's equations directly imply the general differential equation for $\boldsymbol{E}$,

$$
\nabla \times \nabla \times \boldsymbol{E}=k^{2} \boldsymbol{E}-j \omega \mu \boldsymbol{J},
$$

which must be specialized appropriately for the present problem. $\boldsymbol{E}$ is moreover subject to the boundary condition that its tangential components vanish on perfectly conducting surfaces.

In the present problem both the exciting field and the waveguide-obstacle structure are independent of $y$ in the range $0 \overline{<} y \overline{<} b$, and it follows that the whole field, secondary as well as primary, will be independent of $y$ in the same range. Further, since the $x$-and $z$-components of $\boldsymbol{E}$ must vanish in particular on the $y=0$ surface of the waveguide, these components must vanish for all values of $y$ involved. Thus the electric field is of the form $\boldsymbol{E}=\boldsymbol{\phi}(x, z) \boldsymbol{e}_{y}$; eq $(3.1)$ (with $\boldsymbol{J} \equiv 0$ ) reduces to

$$
\nabla^{2} \phi+k^{2} \phi=0 \quad \text { in } S,
$$

and the boundary condition becomes

$$
\phi=0 \quad \text { on } \quad C,
$$

where $S$ is the cross section and $C$ is the boundary of the structure in a plane $y=$ constant (fig. 3 ).
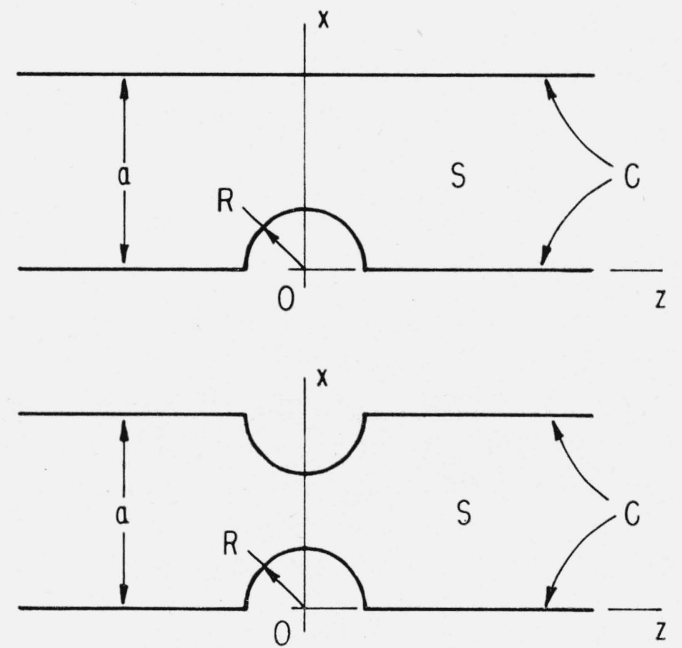

Figure 3. Relevant geometry and coordinates for the single and double half-round problems.

The magnetic field corresponding to $\boldsymbol{E}$ of the above form is

$$
\boldsymbol{H}=(j \omega \mu)^{-1}\left(\frac{\partial \phi}{\partial z} \boldsymbol{e}_{x}-\frac{\partial \phi}{\partial r} \boldsymbol{e}_{z}\right)
$$

The surface current $\boldsymbol{K}=\boldsymbol{H} \times \boldsymbol{n}$ on $C$ is thus given by

$$
\boldsymbol{K}=-(j \omega \mu)^{-1} \boldsymbol{n} \cdot \nabla \phi \boldsymbol{e}_{y}
$$

evaluated on $C$, where $\boldsymbol{n}$ is the outward normal unit vector on $C$. Thus $\boldsymbol{K}$ has only a $y$-component and may be written as $K(x, z) \boldsymbol{e}_{y}$.

Inasmuch as the whole problem can be dealt with in terms of $\phi$ and $K$, the problem is essentially a two-dimensional scalar one. In what follows it will be convenient to refer to $\phi$ simply as "the field". 


\subsection{Integral Equation Formulation}

We now introduce a Green's function $\Gamma=\Gamma\left(x, z, x^{\prime}, z^{\prime}\right)$ such that $\Gamma$ is the field that would be produced in the unperturbed waveguide at the point $(x, z)$ by a unit current filament parallel to $\boldsymbol{e}_{y}$ at the point $\left(x^{\prime}, z^{\prime}\right)$. As a function of $(x, z), \Gamma$ satisfies the differential equation

$$
\nabla^{2} \Gamma+k^{2} \Gamma=j \omega \mu \delta\left(x-x^{\prime}\right) \delta\left(z-z^{\prime}\right)
$$

obtained from (3.1) with $\boldsymbol{J}=\delta\left(x-x^{\prime}\right) \delta\left(z-z^{\prime}\right) \boldsymbol{e}_{y}$, where $\delta$ denotes the Dirac delta-function. $\Gamma$ further satisfies the boundary condition $\Gamma=0$ for $x=0$, $a$ and represents outgoing waves for $\left|z-z^{\prime}\right| \rightarrow \infty$. An explicit form for $\Gamma$ is [5]

$$
\begin{aligned}
\Gamma\left(x, z, x^{\prime}, z^{\prime}\right)=-\omega \mu(\beta a)^{-1} \sin (\pi x / a) \sin \left(\pi x^{\prime} / a\right) e^{-j \beta\left|z-z^{\prime}\right|} & \\
& -(j \omega \mu / a) \sum_{n=2}^{\infty} \alpha_{n}^{-1} \sin (n \pi x / a) \sin \left(n \pi x^{\prime} / a\right) e^{-\alpha_{n}\left|z-z^{\prime}\right|},
\end{aligned}
$$

where $\alpha_{n}=\sqrt{(n \pi / a)^{2}-k^{2}}$ for $n>2$ and the other symbols are as already defined.

It is convenient to put

$$
\Gamma=-\omega \mu(F+j G)
$$

where $F$ and $G$ are real. We observe that $F$ can be written in the form

$$
F=(\beta a)^{-1}\left[\psi_{e}(x, z) \psi_{e}\left(x^{\prime}, z^{\prime}\right)+\psi_{0}(x, z) \psi_{0}\left(x^{\prime}, z^{\prime}\right)\right],
$$

with the definitions

$$
\left.\begin{array}{l}
\psi_{e}(x, z)=\cos \beta z \sin (\pi x / a), \\
\psi_{0}(x, z)=\sin \beta z \sin (\pi x / a) .
\end{array}\right\}
$$

The functions $\psi_{\mathrm{e}}$ and $\psi_{0}$ are respectively symmetric and antisymmetric with respect to reflection in the plane $z=0$.

With the aid of the Green's function we can set up the fundamental equations of the problem in integral-equation form. Let $\psi=\psi(x, z)$ denote the exciting field-i.e., the field, due to remote sources, that would exist in the absence of obstacles. Then, with an obstacle present, the total field $\phi$ can be written as the sum of the exciting field and the secondary field due to the current on the obstacle:

$$
\phi(x, z)=\psi(x, z)+\int_{C_{0}} \Gamma\left(x, z, x^{\prime}, z^{\prime}\right) K\left(x^{\prime}, z^{\prime}\right) d C^{\prime},
$$

where $K$ is the surface current on the obstacle and the integral is a line integral going over the contour $C_{0}$ of the obstacle ( $C_{0}$ consists of two parts in the case of the double half-rounds). If the point $(x, z)$ is taken on $C_{0}, \phi$ must vanish and we have

$$
0=\psi(x, z)+\int_{C_{0}} \Gamma\left(x, z, x^{\prime}, z^{\prime}\right) K\left(x^{\prime}, z^{\prime}\right) d C^{\prime}, \quad(x, z) \text { on } C_{0} .
$$

This is an inhomogeneous integral equation of the first kind determining $K$ for a given $\psi$. The equation reduces to slightly different forms in the symmetric and antisymmetric cases, which we now consider.

$$
\text { a. Symmetric Case }
$$

Let the exciting field be $A \psi_{e}(x, z)$, where $A$ is an arbitrary amplitude and $\psi_{e}$, as defined in (3.6), is a symmetric function of $z$. The surface current on the obstacle will then also be a symmetric function of $z$; we denote it by $K_{\ell}$. We now examine (3.7) for $z<<z^{\prime}$, say, to find the lowest-mode component and thus to find $v_{1, e}(z)$ and $i_{1, e}(z)$. For $z<<z^{\prime}$, (3.7) becomes

$$
\phi(x, z)=A \cos \beta z \sin (\pi x / a)-(\beta a)^{-1} \omega \mu \sin (\pi x / a) e^{j \beta z} \int_{C_{0}} \psi_{e} K_{e} d C,
$$


since integrals of the type $\int_{C_{0}} \psi_{0} K_{e} d C$ vanish because of the mutual orthogonality of symmetric and antisymmetric functions. By comparison with (2.6) it is seen that

$$
v_{1, e}(z)=A \cos \beta z-(\beta a)^{-1} \omega \mu e^{\mathrm{j} \beta z} \int_{C_{0}} \psi_{e} K_{e} d C .
$$

Using (3.2a) to obtain the $x$-component of the magnetic field associated with (3.9) and again referring to (2.6) one finds

$$
i_{1, e}(z)=-j A \sin \beta z+(\beta a)^{-1} \omega \mu e^{j \beta z} \int_{C_{0}} \psi_{e} K_{e} d C .
$$

Equations (3.10) and (3.11a) hold directly from the definitions for $z<-R$ and by extrapolation for $z=0$. In particular (3.11a) yields

$$
i_{1, e}(0)=(\beta a)^{-1} \omega \mu \int_{C_{0}} \psi_{e} K_{e} d C
$$

Next, in the integral eq (3.8), we separate the Green's function into real and imaginary parts as in (3.5), use the orthogonality property of even and odd functions, and thus find

$$
\psi_{e}(x, z) v_{1, e}(0)=j \omega \mu \int_{C_{0}} G\left(x, z, x^{\prime} z^{\prime}\right) K_{e}\left(x^{\prime}, z^{\prime}\right) d C^{\prime} .
$$

This equation and (3.11b) together furnish a definitive mathematical statement of the symmetric part of our problem.

\section{b. Antisymmetric Case}

In this case we let the exciting field be $A \psi_{0}(x, z)$, where $A$ is again an arbitrary constant and $\psi_{0}$, defined in (3.6), is an antisymmetric function of $z$. The current, $K_{0}$, on the obstacle will then also be antisymmetric with respect to $z$. In the same manner as in the symmetric case one finds, for $z<0$,

$$
v_{1,0}(z)=A \sin \beta z+(\beta a)^{-1} j \omega \mu e^{j \beta z} \int_{C_{0}} \psi_{0} K_{0} d C
$$

In particular,

$$
v_{1,0}(0)=(\beta a)^{-1} j \omega \mu \int_{C_{0}} \psi_{0} K_{0} d C
$$

Further,

$$
i_{1,0}(z)=j A \cos \beta z-(\beta a)^{-1} j \omega \mu e^{j \beta z} \int_{C_{0}} \psi_{0} K_{0} d C .
$$

and the integral equation becomes

$$
\psi_{0}(x, z) i_{1,0}(0)=-\omega \mu \int_{C_{0}} G\left(x, z, x^{\prime}, z^{\prime}\right) K_{0}\left(x^{\prime}, z^{\prime}\right) d C^{\prime} .
$$

This equation and (3.14b) together furnish a definitive statement of the antisymmetric cases of our problem.

\subsection{Summary of all Cases}

In the case of the double half-rounds the above integrations go over a two-part contour consisting of an upper semicircle $C_{u}$ and a lower semicircle $C_{l}$. An equivalent problem involving only one of the parts of the contours, say $C_{l}$, can be formulated using the fact that the exciting field as well as the structure is symmetric with respect to the plane $x=a / 2$, so that the current on the obstacles must also be symmetric with respect to this plane. This symmetry is quite 
independent of the symmetries with respect to $z=0$ distinguished by the subscripts " $e$ " and "o," and so these subscripts will be dropped for the moment. Thus, it is clear that

$$
\int_{C_{o}} \psi K d C=\int_{C_{u}} \psi K d C+\int_{C_{l}} \psi K d C=2 \int_{C_{l}} \psi K d C
$$

Further,

$$
\int_{C_{o}} G\left(x, z, x^{\prime}, z^{\prime}\right) K\left(x^{\prime}, z^{\prime}\right) d C^{\prime}=\int_{C_{l}} G^{(2)}\left(x, z, x^{\prime}, z^{\prime}\right) K\left(x^{\prime}, z^{\prime}\right) d C^{\prime},
$$

where $G^{(2)}$ is defined by

$$
G^{(2)}\left(x, z, x^{\prime}, z^{\prime}\right)=G\left(x, z, x^{\prime}, z^{\prime}\right)+G\left(x, z, a-x^{\prime}, z^{\prime}\right) .
$$

Equations (3.18) and (3.19) enable the desired restatement of the double half-round problems.

In what follows, superscripts 1,2 will be used when it is desired to distinguish quantities associated with the single and the double half-round problems, respectively (this means in particular $\left.G \equiv G^{(1)}\right)$. Integrals will be indicated by means of the convenient scalar product notation, e.g.,

$$
(\psi, K)=\int_{C_{l}} \psi K d C
$$

and by the operator notation

$$
G K=\int_{C_{l}} G\left(x, z, x^{\prime}, z^{\prime}\right) K\left(x^{\prime}, z^{\prime}\right) d C^{\prime} .
$$

As indicated, all such integrals are to be taken over $C_{l}$, the lower semicircle. As a further notational convenience, we introduce the "normalized" surface currents,

$$
I_{e}=j \omega \mu K_{e} / v_{1, e}(0), \quad I_{o}=-\omega \mu K_{o} / i_{1, o}(0),
$$

for both the single and the double problems.

Our results thus far may now be summarized as follows. For the symmetric cases we have

$$
\psi_{e}=G^{(h)} I_{e}, \quad 1 / X_{e e}^{(h)}=h\left(\psi_{e}, I_{e}\right) /(\beta a)
$$

and for the antisymmetric cases

$$
\psi_{o}=G^{(h)} I_{o}, \quad X_{o o}^{(h)}=-h\left(\psi_{o}, I_{o}\right) /(\beta a),
$$

where $h=1$ or 2 for the single or the double half-rounds, respectively, and $X_{e e}, X_{o o}$ are the reactances corresponding to the (pure imaginary) impedances $Z_{e e}, Z_{o o}$ defined in (2.9).

\section{Solution for the Reactances}

\subsection{Application of Rayleigh-Ritz Method}

In each of the problems specified by (3.22) and (3.23), an unknown reactance or susceptance is proportional to a scalar product

$$
M=(\psi, I),
$$

where the function $I$ is determined by an integral equation,

$$
G I=\psi,
$$

in which $\psi$ and $G$ are given and $G$ is symmetric. From these equations one may easily construct the "stationary representation" $[1,2]$

$$
M=(\psi, I)^{2} /(I, G I)
$$


for $M$. This constitutes the starting point for the method of Rayleigh and Ritz [6], which we wish to use to obtain an approximate expression for $M$. One assumes in (4.2) an approximation for the surface current in the form

$$
I_{n}=x_{i} f_{i}
$$

where the $x_{i}$ are coefficients to be determined, the $f_{i}$ are members of a suitable set of basis functions (to be chosen explicitly), and summation from 1 to $n$ over repeated indices $i, j$, . ., is understood. Expression (4.2) becomes an ordinary function of the $x_{i}$ and is to be subjected to the conditions of stationarity $\partial M / \partial x_{i}=0, \quad i=1,2, \ldots, n$. This leads to the system of equations

where

$$
G_{i j x_{j}}=\left(x_{i} c_{k} / M_{n}\right) c_{i}
$$

$$
c_{i} \equiv\left(\psi, f_{i}\right), \quad G_{i j} \equiv\left(f_{i}, G f_{j}\right),
$$

and $M_{n}$ denotes the now-determinate approximate value for $M$. If the ar bitrary normalization of the $x_{i}$ is chosen so that $x_{k} c_{k}=M_{n}$, then

$$
x_{i}=\left(G^{-1}\right)_{i j} c_{j}
$$

where $\left(G^{-1}\right)_{i j}$ denotes an element of the inverse of the $n \times n$ matrix of the $G_{i j}$, and it immediately follows that

$$
M_{n}=\left(G^{-1}\right)_{i j} c_{i} c_{j}
$$

Finally, we write this quadratic form as a ratio of determinants,

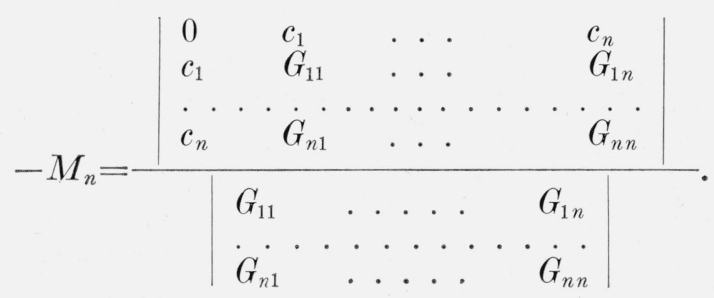

Thus $M_{n}$ is expressed in terms of the known quantities (4.4); if the objective is only to calculate the reactances, it is not necessary to calculate the $x_{i}$.

\subsection{Definiteness of $G$; Upper Bounds for $X_{00}$}

Although in our problem $G$ is not a definite kernel, it does have definite character with respect to a suitably restricted class of admissible functions. In fact, $G$ is positive definite with respect to functions that are antisymmetric with respect to $z$ and vanish for $|z|>\lambda_{g} / 4$, where $\lambda_{g}=2 \pi / \beta$ is the "guide wavelength" of the single propagating mode. ${ }^{3}$ In terms of the integrals of interest here, this means that if $\tilde{I}_{0}$ is any assumed surface current distribution, continuous, not identically equal to zero, and antisymmetric with respect to $z$, then

provided $R<\lambda_{g} / 4$.

$$
\left(\tilde{I}_{0}, G \tilde{I}_{0}\right)>0
$$

Now, for a positive definite kernel it is well known that the right-hand side of (4.2) is not only stationary, but also a maximum for the true $I$. For this result we offer the following brief proof based directly on the Schwarz inequality. ${ }^{4}$ Assuming $G$ to be positive definite, we wish to compare the approximate value for $M$ given by

$$
\tilde{M}=(\tilde{I}, \psi)^{2} /(\tilde{I}, G \tilde{I})
$$

3 This result is suggested by qualitative physical considerations and has been verified analytically by the author. It has also been verified that $G$ is not definite with respect to functions symmetric with respect to $z$.

${ }_{4}^{4}$ Proofs of this result in the literature known to the author, e.g., [1,2], employ the so-called bilinear expansion for the kernel involved and do not yield the "only if" part of the result (4.7). 
where $\tilde{I}$ is an admissible approximation for the surface current, with the true value given by (4.1a) (as well as by (4.2)) when $\tilde{I}$ satisfies (4.1b). From $(4.1 \mathrm{~b})$ it follows that $(\tilde{I}, \psi)=(\tilde{I}, G I)$; the Schwarz inequality for positive-definite symmetric transformations [7] gives

$$
(\tilde{I}, G I)^{2} \overline{<}(\tilde{I}, G \tilde{I})(I, G I) .
$$

Since $(I, G I)=(I, \psi)=M$, we have the result

$$
\tilde{M} \overline{<} M
$$

Moreover the sign of equality holds in the Schwarz inequality and hence in this result if and only if $\tilde{I}$ is proportional to $I$.

Clearly, (4.7) is applicable when $G$ is merely positive definite with respect to all functions that need be admitted; by (4.6) this will be the case in the antisymmetric parts of our problem provided $R<\lambda_{g} / 4$. Hence, subject to this inequality, in the antisymmetric cases we shall have $M_{n}<M$ (we do not anticipate an exact result for finite $n$ ); this in turn implies that numerical values for $X_{00}$ will be (algebraic) upper bounds for the true values - assuming, of course, that numerical evaluations involved are sufficiently precise.

Unfortunately one can not obtain a bound for $X_{e e}$ in this simple manner. Furthermore, derived quantities of practical interest (such as (4.17)) will usually depend upon both $X_{e e}$ and $X_{00}$. Thus in general no bounds for such derived quantities are determined.

\subsection{Basis Functions}

We introduce plane polar coordinates $r, \theta$ such that

$$
x=r \sin \theta, \quad z=r \cos \theta .
$$

The path of integration $C_{l}$ is then given parametrically by

$$
x=R \sin \theta, \quad z=R \cos \theta,
$$

where $R$ is the obstacle radius and $0 \overline{<} \theta \overline{<} \pi$. On this path the surface current $I=I(\mathrm{R} \sin \theta$, $R \cos \theta$ ) becomes a function of $\theta$, which we denote simply by $I(\theta)$. We observe that symmetries with respect to $z=0$ are equivalent to symmetries with respect to $\theta=\pi / 2$.

As basis functions we take

$$
f_{i}=2(\pi R)^{-1} \sin (2 i-1) \theta
$$

for the symmetric cases (expansion of $I_{\varepsilon}$ ), and

$$
f_{i}=2(\pi R)^{-1} \sin 2 i \theta
$$

for the antisymmetric cases (expansion of $I_{0}$ ). The two sets of functions are complete for the expansion of symmetric and antisymmetric functions, respectively, in the interval $(0, \pi)$. The completeness gives good assurance that for sufficiently large $n, M_{n}$ will approach arbitrarily close to the true $M$. For practical numerical calculation it is vital that the convergence of $M_{n}$ be rapid. In the present instance rapid convergence might be anticipated on the grounds that the true $I_{e}$ and $I_{0}$ may be expected to be smooth (possibly infinitely smooth) functions of $\theta$ vanishing at 0 and $\pi$, so that their Fourier sine series should converge rapidly. ${ }^{5}$

\footnotetext{
5 The points $(R, 0)$ and $(R, \pi)$ are the vertices of the corners formed where the half-round centered at $O$ meets the waveguide wall. The vanishing of the surface current in these vertices is equivalent, by (3.2), to the vanishing of the components of the surface magnetic field normal to the vertices. That these field components should vanish in the limit as a vertex is approached follows from properties of Sommerfeld's solution [14] of the problem of diffraction by a wedge of arbitrary angle.
} 


\subsection{Evaluation of Integrals}

Integrals of the type $\left(\psi, f_{i}\right)$ may be obtained relatively easily with the aid of the well-known expansion [8]

$$
e^{j \zeta \sin \alpha}=\sum_{n=-\infty}^{\infty} J_{n}(\zeta) e^{j n \alpha},
$$

wherein $J_{n}$ is the $n^{\text {th }}$ order Bessel function of the first kind. In this expansion we put $\zeta=k R$ and $\alpha= \pm \theta-\chi+(\pi / 2)$, with $k \sin \chi=\pi / a, \quad k \cos \chi=\beta$, and employ (4.9). The expansion

$$
e^{j \beta z} \sin (\pi x / a)=2 \sum_{n=1}^{\infty} j^{n-1} J_{n}(k R) \sin n \theta \sin n \chi
$$

is then easily obtained. The integrations yielding the desired quantities are now elementary; one obtains

$$
c_{i}=2(-)^{(p-1) / 2} J_{p}(k R) \sin p \chi, \quad p=2 i--1,
$$

for the symmetric cases, and

$$
c_{i}=2(-)^{(p-2) / 2} J_{p}(k R) \sin p \chi, \quad p=2 i,
$$

for the antisymmetric cases. (It is encouraging that these quantities decrease rapidly with increasing index.)

The evaluation of the integrals of the type $\left(f_{i}, G f_{j}\right)=G_{i j}$ is considerably more complicated than the evaluation of the $c_{i}$, and the task has been relegated to the appendix The results obtained may be summarized conveniently in the following form, which comprehends four cases:

$$
\frac{G_{j j}^{(h)}}{c_{i} c_{j}}=-\frac{1}{4 s_{p} s_{q}}\left[\rho_{p}(k R) \delta_{p q}+\sigma_{p q}^{(h)}(k a)\right]
$$

Here $s_{p} \equiv \sin p \chi ; \rho_{p}(k R) \equiv Y_{p}(k R) / J_{p}(k R)$; in the symmetric cases, $p=2 i-1$ and $q=2 j-1$; in the antisymmetric cases, $p=2 i$ and $q=2 j$; the functions $\sigma_{p q}^{(h)}$ are defined in the appendix (and tabulated briefly in table 2); $Y_{p}$ denotes the Bessel function of the second kind; and all other symbols involved have been defined previously.

\subsection{Results}

The value of a reactance element corresponding to an $n$-term expansion (4.3) will be called an " $n^{\text {th }}$ approximation" and will be written simply $X_{e e}^{(h)}$ or $X_{00}^{(h)}$ without special notation indicating the value of $n$ being considered. An explicit expression, in determinantal form, for $X_{e e}^{(h)}$ in the $n^{\text {th }}$ approximation may be obtained by combining (3.22), (4.5), and (4.14); viz,

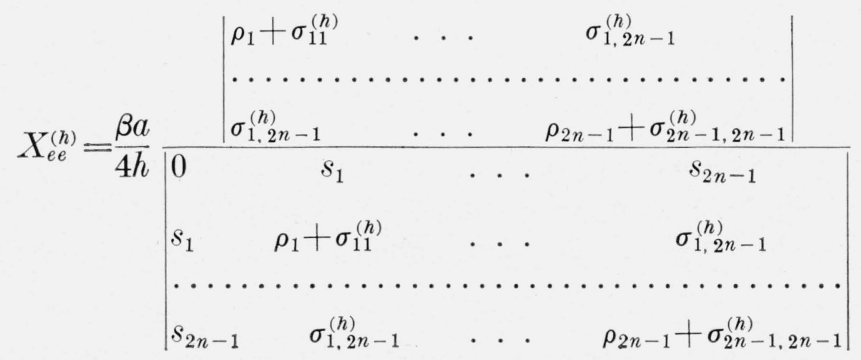


(In this and in the following equation the arguments $k R$ and $k a$ are understood.) Similarly, $(3.23),(4.5)$, and (4.14) yield

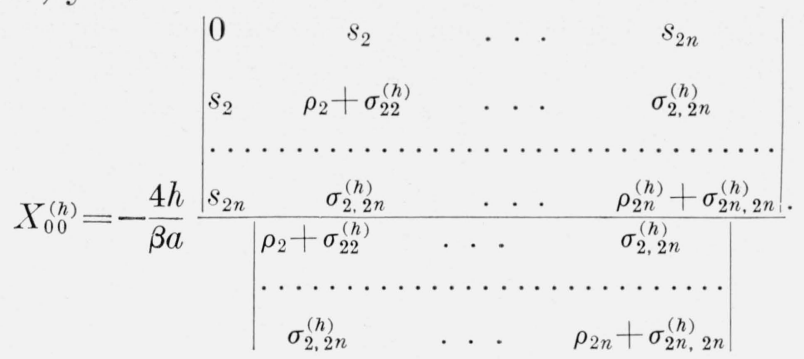

The determinantal form of these expressions seems convenient enough for practical calculations with $n$ at least as large as 3 . However, it may be remarked that, because of the special form of the diagonal elements, the determinants can be expanded in the same way as certain determinants occurring in the Fredholm theory of integral equations. This expansion may be convenient for the consideration of arbitrarily large $n$, but is not needed here.

Once the reactances $X_{e e}$ and $X_{00}$ are determined, other quantities or parameters associated with the representation of an obstacle are of course also determined. In particular, the "voltage" standing-wave ratio seen on one side of an obstacle when the other side is terminated in a matched (reflectionless) load is a familiar quantity having immediate physical significance, and it is convenient to discuss some of the results in terms of this quantity. Recalling that waveguide characteristic impedances were chosen equal to unity and referring to the equivalent network, figure 2, it is easily found that the standing-wave ratio in question is given by

$$
\eta=(1+|S|) /(1-|S|),
$$

where $|S|$, the magnitude of the associated reflection coefficient, is

$$
|S|=\left[1+\left(\frac{X_{e e}-X_{00}}{X_{e e} X_{00}+1}\right)^{2}\right]^{-1 / 2} \text {. }
$$

In order to get some idea of the behavior of the sequence of approximations, the reactance elements and the related VSWR (4.17) have been calculated for $n=1,2$, and 3 and for a range of values of $k a$ and $k R$. Table 1 presents more or less typical results in terms of the values of VSWR. The convergence of the sequence of approximations, judged on the basis of numerical results exemplified in the table, appears to be very rapid; by the same token, the first approximation furnishes a rather good result for VSWR's up to about 2.

Thus far it has not proved feasible to determine the manner of convergence of the sequence of approximations by theoretical means. In view of the character of the particular problem at hand, it seems probable that the numerical evidence may be relied upon.

TABLE 1. Convergence of VSWR

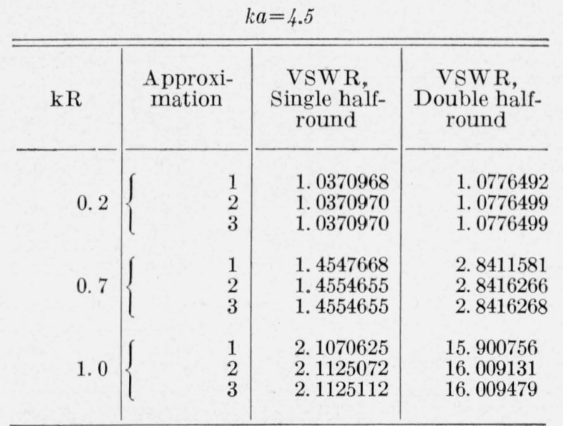

Note: Maximum computational inaccuracy in the above figures is estimated to be approximately \pm 1 in the sixth significant figure; eight figures, as given, may be significant in indicating the behavior of successive approximations. 
Further calculations have been made regarding $k a$ and $R / a$ as independent variables. This choice of variables corresponds to the practical situation where one has a given obstacle (having a given value of $R / a$ ) that is to be operated at various frequencies. Figures 4 and 5 show the matched-termination VSWR (4.17) resulting for various values of $R / a$ and $f / f_{c}$, where $f$ is the operating frequency and $f_{c}$ is the cut-off frequency for the $T E_{10}$ mode (and $\left.f / f_{c}=k a / \pi\right)$. (Precise tables (based on the third approximations) giving basic parameters and possibly some derived quantities are being prepared for separate publication.)

The first approximations appear to be of useful accuracy for some purposes and represent an appreciable extension of previously available results. Consequently, a brief tabulation of the $\sigma$-functions, sufficient for the calculation of first approximations, is presented as table 2 . For convenience of reference we write down (4.15) and (4.16) for the special case $n=1$,

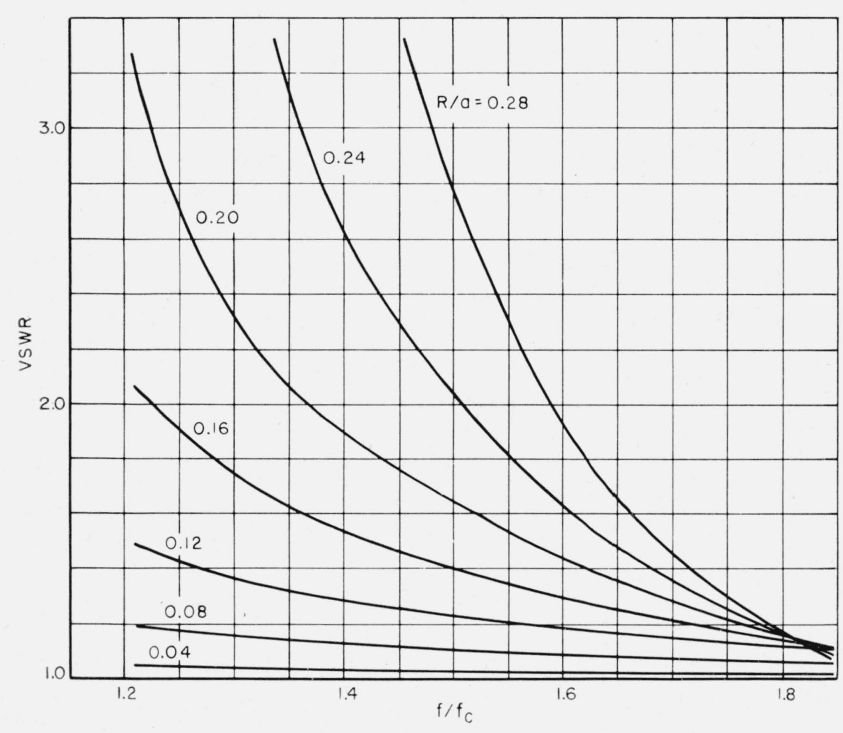

Figure 4. VSWR versus $f / f_{c}$ for single half-rounds.

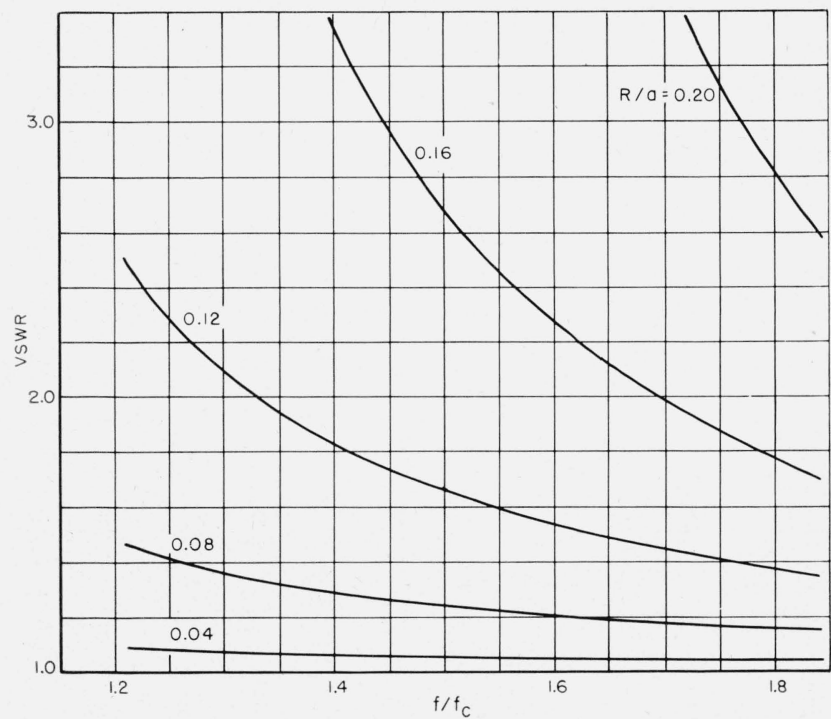

Figure 5. VSWR versus $f / f_{c}$ for double half-rounds.

$$
\begin{aligned}
& X_{e e}^{(h)}=-\frac{\beta a}{4 h s_{1}^{2}}\left[\rho_{1}(k R)+\sigma_{11}^{(h)}(k a)\right], \\
& X_{00}^{(h)}=\frac{4 h s_{2}^{2}}{\beta a}\left[\rho_{2}(k R)+\sigma_{22}^{(h)}(k a)\right]^{-1},
\end{aligned}
$$

and note that $s_{1}=\pi /(k a)$ and $s_{2}=2 \pi \beta /\left(k^{2} a\right)$.

\begin{tabular}{|c|c|c|c|c|}
\hline$x$ & $\sigma_{11}^{(1)}(x)$ & $\sigma_{22}^{(1)}(x)$ & $\sigma_{11}^{(2)}(x)$ & $\sigma_{22}^{(2)}(x)$ \\
\hline $\begin{array}{l}3.80 \\
3.85 \\
3.90 \\
3.95\end{array}$ & $\begin{array}{l}2.055435 \\
1.976710 \\
1.899606 \\
1.823995\end{array}$ & $\begin{array}{c}-0.3170852 \\
-.1982432 \\
-.09078960 \\
006197600\end{array}$ & $\begin{array}{l}3.960997 \\
3.857142 \\
3.756554 \\
3.659060\end{array}$ & $\begin{array}{l}-2.544410 \\
-2.215670 \\
-1.912736 \\
-1.633535\end{array}$ \\
\hline $\begin{array}{l}\text { 4. } 00 \\
4.05 \\
4.10 \\
4.15\end{array}$ & $\begin{array}{l}1.749764 \\
1.676799 \\
1.604988 \\
1.534222\end{array}$ & $\begin{array}{l}.09356160 \\
.1720644 \\
.2424036 \\
.3052036\end{array}$ & $\begin{array}{l}3.564510 \\
3.472757 \\
3.383659 \\
3.297087\end{array}$ & $\begin{array}{l}-1.376168 \\
-1.138910 \\
-0.9201812 \\
-.7185580\end{array}$ \\
\hline $\begin{array}{l}4.20 \\
4.25 \\
4.30 \\
4.35\end{array}$ & $\begin{array}{l}\text { 1. } 464400 \\
\text { 1. } 395414 \\
1.327159 \\
1.259533\end{array}$ & $\begin{array}{l}.3610476 \\
.4104564 \\
.4539056 \\
.4918312\end{array}$ & $\begin{array}{l}\text { 3. } 212923 \\
\text { 3. } 131048 \\
\text { 3. } 051353 \\
\text { 2. } 973735\end{array}$ & $\begin{array}{l}-.5327088 \\
-.3614372 \\
-.2036444 \\
-.05831720\end{array}$ \\
\hline $\begin{array}{l}4.40 \\
4.45 \\
4.50 \\
4.55\end{array}$ & $\begin{array}{l}1.192430 \\
1.125746 \\
1.059372 \\
0.9931968\end{array}$ & $\begin{array}{l}.5246272 \\
.5526568 \\
.5762488 \\
.5956964\end{array}$ & $\begin{array}{l}2.898094 \\
2.824341 \\
2.752387 \\
2.682146\end{array}$ & $\begin{array}{l}.07546600 \\
.1985600 \\
.3117472 \\
.4157360\end{array}$ \\
\hline $\begin{array}{l}4.60 \\
4.65 \\
4.70 \\
4.75\end{array}$ & $\begin{array}{l}.9271072 \\
.8609836 \\
.7946988 \\
.7281208\end{array}$ & $\begin{array}{l}.6112796 \\
.6232416 \\
.6318024 \\
.6371680\end{array}$ & $\begin{array}{l}2.613542 \\
2.546500 \\
2.480946 \\
2.416812\end{array}$ & $\begin{array}{l}.5112028 \\
.5987500 \\
.6789328 \\
.7522756\end{array}$ \\
\hline $\begin{array}{l}4.80 \\
4.85 \\
4.90 \\
4.95\end{array}$ & $\begin{array}{l}.6611076 \\
.5935064 \\
.52511500 \\
.4558572\end{array}$ & $\begin{array}{l}.6395192 \\
.6390196 \\
.6358108 \\
.6300236\end{array}$ & $\begin{array}{l}\text { 2. } 354034 \\
\text { 2. } 292551 \\
\text { 2. } 2323231 \\
\text { 2. } 173228\end{array}$ & $\begin{array}{r}.8192564 \\
.8803180 \\
.9358596 \\
.9862656\end{array}$ \\
\hline $\begin{array}{l}5.00 \\
5.05 \\
5.10 \\
5.15\end{array}$ & $\begin{array}{l}.3854276 \\
.3136376 \\
.2402376 \\
.1649416\end{array}$ & $\begin{array}{l}.6217692 \\
.6111440 \\
.5982284 \\
.5830836\end{array}$ & $\begin{array}{l}\text { 2. } 115279 \\
\text { 2. } 058400 \\
\text { 2. } 002545 \\
1.947661\end{array}$ & $\begin{array}{l}\text { 1. } 031884 \\
\text { 1. } 073038 \\
1.110027 \\
1.143125\end{array}$ \\
\hline $\begin{array}{l}5.20 \\
5.25 \\
5.30 \\
5.35\end{array}$ & $\begin{array}{l}.08742720 \\
.007320000 \\
-.07581400 \\
-.1624856\end{array}$ & $\begin{array}{l}.5657628 \\
.5462944 \\
.5246972 \\
.5009664\end{array}$ & $\begin{array}{l}1.893705 \\
1.840631 \\
\text { 1. } 788397 \\
1.736963\end{array}$ & $\begin{array}{l}1.172594 \\
1.198667 \\
1.221571 \\
1.241508\end{array}$ \\
\hline $\begin{array}{l}\text { 5. } 40 \\
\text { 5. } 45 \\
5.50 \\
\text { 5. } 55\end{array}$ & $\begin{array}{l}-.2533004 \\
-.3489884 \\
-.4504328 \\
-.4587224\end{array}$ & $\begin{array}{r}.4750804 \\
.4469912 \\
.4166292 \\
.3838912\end{array}$ & $\begin{array}{l}\text { 1. } 6862888 \\
\text { 1. } 636332 \\
\text { 1. } 587059 \\
\text { 1. } 538434\end{array}$ & $\begin{array}{l}\text { 1. } 258672 \\
\text { 1. } 273236 \\
\text { 1. } 285370 \\
\text { 1. } 295225\end{array}$ \\
\hline $\begin{array}{l}\text { 5. } 60 \\
5.65 \\
5.70 \\
5.75\end{array}$ & $\begin{array}{l}-.6752200 \\
-.8016548 \\
-.9402632 \\
-1.094004\end{array}$ & $\begin{array}{l}.3486376 \\
.3106864 \\
.2697956 \\
.2256488\end{array}$ & $\begin{array}{l}1.490420 \\
1.442981 \\
1.396088 \\
1.349701\end{array}$ & $\begin{array}{l}1.302940 \\
1.308654 \\
1.312488 \\
1.314555\end{array}$ \\
\hline 5. 80 & -1.266887 & .1778340 & 1. 303798 & 1. 314964 \\
\hline
\end{tabular}

TABLE 2. Values of $\sigma_{p p}^{(h)}(x)$

Note: Accuracy of the above figures is estimated to be approximately one part per million. 
For sufficiently small $k R$, the expressions just given may be simplified still further. For small $k R$, the quantities $\rho_{1}(k R)$ and $\rho_{2}(k R)$ become large, in accordance with the formula

$$
\rho_{q}(k R) \cong-\frac{1}{\pi} q !(q-1) !\left(\frac{2}{k R}\right)^{2 q} \quad(q>1),
$$

and thus determine the qualitative behavior of $X_{e e}$ and $X_{00}$. Using this approximation for the $\rho$ 's, neglecting the $\sigma$ 's, and introducing the guide wavelength $\lambda_{g}=2 \pi / \beta$, one obtains

$$
\begin{aligned}
X_{e e}^{(h)} & =\frac{2 a}{h \lambda_{g}}\left(\frac{a}{\pi R}\right)^{2}, \\
X_{00}^{(h)} & =-\frac{h a}{\lambda_{g}}\left(\frac{\pi R}{a}\right)^{4} .
\end{aligned}
$$

(These are the correct forms of the results in the order of approximation of the more general formulas in the Waveguide Handbook [3].) Although these formulas give a rather good picture of the behavior of the reactances, they fail to reflect the interesting qualitative feature that $X_{e e}^{(h)}$ and $X_{00}^{(h)}$ must eventually become equal to each other as $R$ approaches $a / h$ (as can be seen physically).

\section{Appendix}

\subsection{Expansion of Green's Function in Terms of Cylindrical Wave Functions}

In order to arrive conveniently at the respective types of Green's functions needed for the single and the double half-round problems, we consider a waveguide of width $w$ (instead of $a$ ), bounded by the lines $x=0$ and $x=w$; and, in addition to the electric boundary condition at $x=0$, we consider both electric and magnetic boundary conditions at $x=w$ (by "electric" or "magnetic" boundary conditions it is meant that the Green's function or its normal derivative, respectively, vanishes on the boundary). The radius vectors of the source-point and the field-point will be denoted by $\boldsymbol{r}^{\prime}$ and $\boldsymbol{r}$, respectively.

Now, it is well known that the two-dimensional "free-space" Green's function, satisfying (3.3) and representing outgoing waves at infinity, is $-(\omega \mu / 4) H_{0}\left(k\left|\boldsymbol{r}^{\prime}-\boldsymbol{r}\right|\right)$, where $H_{0}$ denotes the Hankel function of the second kind (as is appropriate for exp ( $j \omega t$ ) time dependence). It is also well known that the desired Green's function, $\Phi$, satisfying (3.3) and the required boundary conditions at $x=0, x=w$, and at $z= \pm \infty$, can be obtained in the form of a sum of free-space Green's functions by the method of images [9]. In fact

$$
\Phi=-(\omega \mu / 4) \sum_{n=-\infty}^{\infty} s^{n}\left[H_{0}\left(k\left|\boldsymbol{\rho}_{n}^{+}-\boldsymbol{r}\right|\right)-H_{0}\left(k\left|\boldsymbol{\rho}_{n}^{-}-\boldsymbol{r}\right|\right)\right],
$$

where $\boldsymbol{\rho}_{n}^{ \pm}=\left(2 n w \pm x^{\prime}\right) \boldsymbol{e}_{x}+z^{\prime} \boldsymbol{e}_{z}$ and $s=+1$ or -1 according to whether the boundary condition at $x=w$ is electric or magnetic. By inspection of the array of sources (fig. 6) it can be seen that this function has the proper symmetry to satisfy the boundary conditions: it is antisymmetric with respect to $x=0$ and it is antisymmetric or symmetric with respect to $x=w$ according to whether $s=+1$ or -1 .

Equation (5.1) can be transformed into a sum of elementary wave-functions of polar coordinates by repeated application of Graf's addition theorem for Bessel functions [10]. We introduce polar coordinates $(r, \theta),\left(r^{\prime}, \theta^{\prime}\right)$, and $\left(\rho_{n}, \theta_{n}\right)$ for $\boldsymbol{r}, \boldsymbol{r}^{\prime}$, and $\boldsymbol{\rho}_{n}$ in the manner shown in (4.8), and we assume $r$ and $r^{\prime}$ both to be less than $w$, so that $\rho_{n}>w$ for $n \neq 0$. Considering the triangles with sides $\boldsymbol{\rho}_{n}, \boldsymbol{r}$, and $\boldsymbol{\rho}_{n}-\boldsymbol{r}$ and applying the addition theorem, we obtain

$$
\begin{aligned}
-4(\omega \mu)^{-1} \Phi=\sum_{p=-\infty}^{\infty} \frac{J_{p}(k r) H_{p}\left(k r^{\prime}\right)}{J_{p}\left(k r^{\prime}\right) H_{p}(k r)} e^{j p \theta}\left(e^{-j p \theta^{\prime}}-e^{j p \theta^{\prime}}\right) & \\
& +\sum_{n=-\infty}^{\infty} s^{n} \sum_{p=-\infty}^{\infty} J_{p}(k r) e^{j p \theta}\left[H_{p}\left(k \rho_{n}^{+}\right) e^{-j \theta+}-H_{p}\left(k \rho_{n}^{-}\right) e^{-j p \theta-}{ }_{n}\right],
\end{aligned}
$$




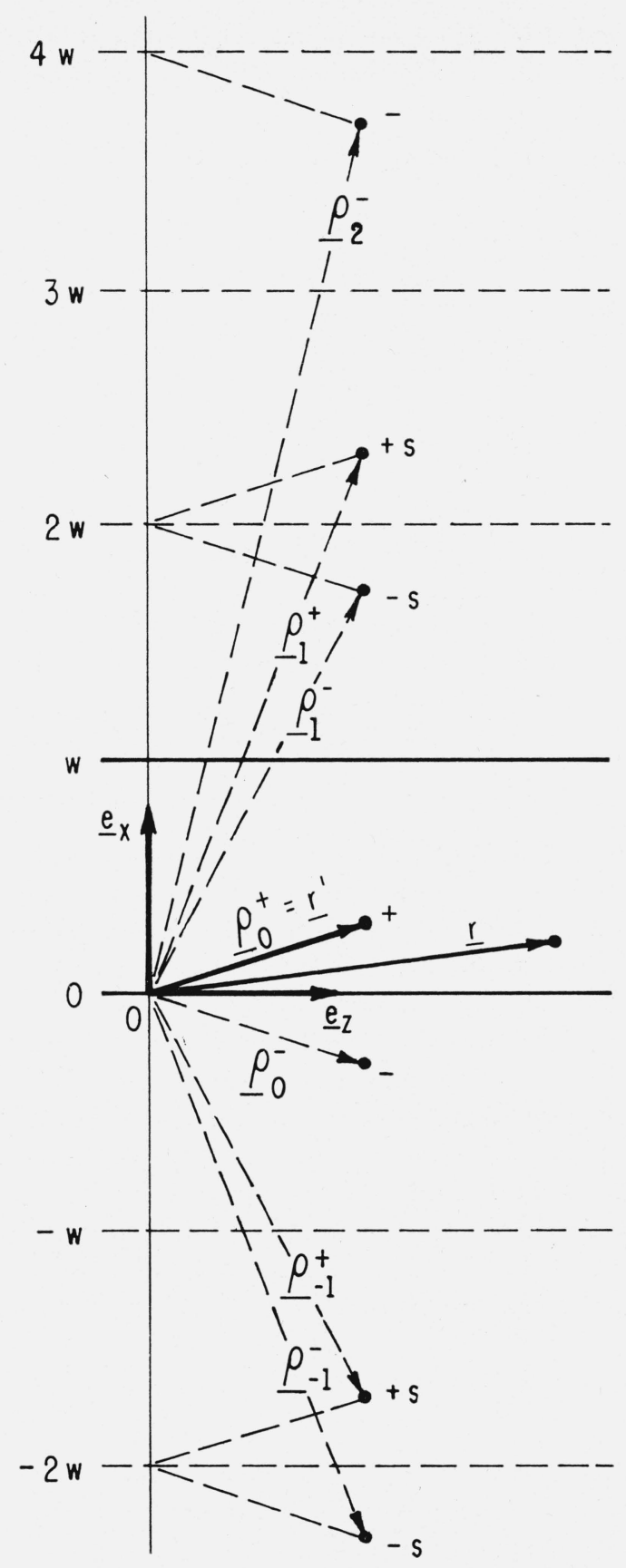

FigueE 6. Source points in the method of images.

Relative signs of sources are indicated by the adjacent symbols,+- ,

$+s$, and $-s$; the role of $s$ is defined in connection with (5.1).

where the terms arising from $n=0$ appear in the first line and are to be omitted from the primed summation in the second line; the upper and lower alternatives in the first line apply according as $r<r^{\prime}$ or $r>r^{\prime}$, respectively. Again, by the addition theorem,

$$
H_{p}\left(k \rho \rho_{n}^{ \pm}\right) e^{-j p \theta_{n}^{ \pm}}=\sum_{q=-\infty}^{\infty} J_{q}\left(k r^{\prime}\right) H_{p+q}(|2 n k w|) \exp \left[j(\pi / 2)(q-p) \operatorname{sgn} n \pm j q \theta^{\prime}\right] .
$$


Combining the last two equations and rearranging terms, one finds that (5.1) can be written in the form

$$
\begin{aligned}
\Phi=-\omega \mu \sum_{p=1}^{\infty} \sum_{q=1}^{\infty} \sin p \theta \sin q \theta^{\prime}\left\{\begin{array}{l}
J_{p}(k r) H_{p}\left(k r^{\prime}\right) \\
J_{p}\left(k r^{\prime}\right) H_{p}(k r)
\end{array} \delta_{p q}\right. \\
\left.+2 J_{p}(k r) J_{q}\left(k r^{\prime}\right) \cos [(\pi / 2)(p-q)] \sum_{n=1}^{\infty} s^{n}\left[H_{p-q}(2 n k w)-H_{p+q}(2 n k w)\right]\right\} .
\end{aligned}
$$

If now one puts $w=a$ and $s=+1$, this expression becomes the desired representation of $\Gamma$ of the text; similarly, if $w=a / 2$ and $s=-1$, (5.2) becomes the desired representation of a Green's function $\Gamma^{(2)}$ related to $G^{(2)}$ in the same way as $\Gamma$ is related to $G$ (cf. 3.5). Thus, employing the relation (3.5), the relation $H_{p}=J_{p}-j Y_{p}$, the notation $(3.20)$, the basis functions $(4.10,4.11)$, and carrying out the integrations with respect to $\theta, \theta^{\prime}$, one obtains

$$
\left(\frac{2}{\pi R}\right)^{2}\left(\sin \mathrm{p} \theta, G^{(h)} \sin q \theta^{\prime}\right)=-J_{p}(k R) Y_{p}(k R) \delta_{p g}-J_{p}(k R) J_{q}(k R) \cos [(\pi / 2)(p-q)] \sigma_{p q}^{(h)}(k a),
$$

where the functions $\sigma_{p q}^{(h)}$ are defined as

$$
\begin{aligned}
& \sigma_{p q}^{(1)}(k a)=2 \sum_{n=1}^{\infty}\left[Y_{p-q}(2 n k a)-Y_{p+q}(2 n k a)\right], \\
& \sigma_{p q}^{(2)}(k a)=2 \sum_{n=1}^{\infty}(-)^{n}\left[Y_{p-\vartheta}(n k a)-Y_{p+q}(n k a)\right] .
\end{aligned}
$$

We note that the above expressions are needed only for positive integer indices $p, q$ that are both even or both odd. We observe also that the form of the expressions is such that those with unequal subscript indices can be evaluated simply in terms of those with equal subscript indices. Further, in view of the easily-verified relation

$$
\sigma_{p q}^{(2)}(k a)=2 \sigma_{p q}^{(1)}(k a)-\sigma_{p q}^{(1)}(k a / 2),
$$

values or formulas for the $\sigma$ 's "of the second kind" can be derived readily from the same for the $\sigma$ 's "of the first kind." Thus, the evaluation of the $G_{p q}^{(h)}$, which, on the face of it, originally involved double integrals with $k a, k R, p, q$, and $h$ as independent parameters, has been reduced essentially to the evaluation of sums of the form $\sum_{n=1}^{\infty} Y_{2 s}(n x)$ with $\pi<x<2 \pi$ or $2 \pi<x<4 \pi$ and $s=0,1,2, \ldots$. It should be observed, however, that these sums converge extremely slowly: for the accuracy desired in the present circumstances the number of terms required would be of the order of $10^{10}$ or more, at least for the most important $\sigma$ 's. Needless to say, some method of evaluation other than direct summation is indicated.

\subsection{Evaluation of the Bessel-Function Series}

Two methods of evaluating the series defined in (5.4) will be discussed briefly here.

\section{a. Conversion to Definite Integrals}

An evaluation of the desired series may be obtained in terms of definite integrals as follows. We start with the series of Hankel functions (of the second kind)

$$
S_{\lambda}(x)=\sum_{n=1}^{\infty} H_{\lambda}(n x)
$$

where $x$ is real, positive, and not an integer multiple of $2 \pi$. For the Hankel functions we use the integral representation [11]

$$
H_{\lambda}(x)=\frac{1}{\pi} \int_{C} e^{-j x \sin \zeta+j \lambda \zeta} d \zeta
$$


where $\zeta=\xi+j \eta$ and $C$ is a suitable path, to be chosen in a moment, between the limits $-j \infty$ and $\pi+j \infty$. With the aid of the well-known expression for the sum of a finite number of terms of a geometric series, one finds that the $M$-term partial sum of (5.6) can be written

$$
\sum_{n=1}^{M} H_{\lambda}(n x)=\frac{1}{\pi} \int_{C} \frac{e^{j \lambda \zeta} d \zeta}{e^{j x \sin \zeta}-1}-\frac{1}{\pi} \int_{C} \frac{e^{-j M x \sin \zeta}}{e^{j x \sin \zeta}-1} e^{j \lambda \zeta} d \zeta
$$

Among the infinitely many poles of the integrands in these integrals, we note in particular $\xi_{m}^{\prime}$ ! and $\xi_{m}^{\prime \prime}=\pi-\xi_{m}^{\prime}$, where $\xi_{m}^{\prime}$ is the principal value of $\sin ^{-1}(2 m \pi / x), m=0,1,2, \ldots, Q$, and $Q$ is the largest integer such that $2 Q \pi / x<1$. The path $C$ is now chosen to comprise (except for indentations) the negative imaginary axis, the real axis between 0 and $\pi$, and the upper half of the line $\pi+j \eta$; indentations, of radius $\rho$, are made at each of the above identified poles in the manner shown in figure 7, which illustrates $C$ for the case $Q=1$. Taken on this path, the second integral in (5.7) approaches 0 as $M$ increases indefinitely; as a detailed examination shows, this happens because on the various parts of the path the integrand approaches 0 or it becomes increasingly oscillatory (or both) as $M \rightarrow \infty$. Hence we have

$$
S_{\lambda}(x)=\frac{1}{\pi} \int_{C} \frac{e^{j \lambda \zeta} d \zeta}{e^{j x \sin \zeta}-1} \cdot
$$

This result can be expressed in terms of real integrations plus contributions from the indentations. In the limit as $\rho \rightarrow 0$, the indentations yield

$$
R_{o}^{\prime}=\frac{1}{2 x}, \quad R_{o}^{\prime \prime}=\frac{e^{j \lambda \pi}}{2 x}
$$

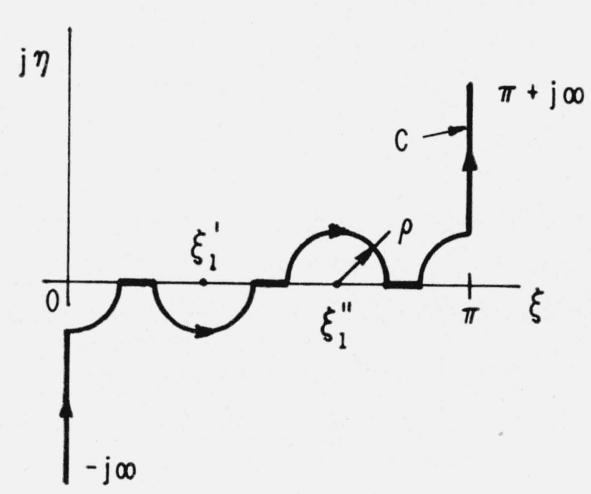

Figure 7. Path of integration.

and for $m>1$,

$$
R_{m}^{\prime}=\frac{e^{j \lambda \xi_{m}^{\prime}}}{\sqrt{x^{2}-(2 m \pi)^{2}}}, \quad R_{m}^{\prime \prime}=\frac{e^{j \lambda\left(\pi-\xi_{m}^{\prime}\right)}}{\sqrt{x^{2}-(2 m \pi)^{2}}} \cdot
$$

Here the notation corresponds to that used for the respective poles and the factor $1 / \pi$ standing in front of the integral is included. Provided $\lambda$ is an even integer, the straight-line segments of the path of integration yield"

$$
-\frac{1}{2} \delta_{o \lambda}+\lim _{\rho \rightarrow 0} \frac{j}{\pi}\left[2 \int_{\rho}^{\infty} \frac{\cosh \lambda \eta d \eta}{e^{x \sinh \eta}-1}-P \int_{\rho}^{\pi / 2} \cos \bar{\eta} \lambda \xi \cot \left(\frac{1}{2} x \sin \xi\right) d \xi\right]
$$

where $\delta_{0 \lambda}$ is the Kronecker delta. It should be noted that when the integrand in the finite integral is singular, the Cauchy principal value is to be taken, as indicated by the symbol $P$ (the indicated limit as $\rho \rightarrow 0$ essentially is also a Cauchy value).

Upon comparing (5.4) and (5.6) it is seen that

$$
\sigma_{p p}^{(1)}(k a)=2 \operatorname{Im}\left[S_{2 p}(2 k a)-S_{0}(2 k a)\right] .
$$


It happens that for $\lambda=2 p$ (where $p=0,1,2, \ldots$ ) the net imaginary contribution of (5.8) to $S_{2 p}$ vanishes. Consequently (5.9) yields

$$
\sigma_{p p}^{(1)}(k a)=\frac{8}{\pi} \int_{0}^{\infty} \frac{\sinh ^{2} p \eta d \eta}{e^{2 k a \sinh \eta}-1}+\frac{4}{\pi} P \int_{0}^{\pi / 2} \sin ^{2} p \xi \cot (k a \sin \xi) d \xi
$$

wherein the integrands are now well-behaved at their lower limits and thus we were permitted to put $\rho=0$. The corresponding result for $\sigma_{p p}^{(2)}$ is

$$
\sigma_{p p}^{(2)}(k a)=-\frac{8}{\pi} \int_{0}^{\infty} \frac{\sinh ^{2} p \eta d \eta}{e^{k a \sinh \eta}+1}-\frac{4}{\pi} P \int_{0}^{\pi / 2} \sin ^{2} p \xi \tan \left(\frac{k a}{2} \sin \xi\right) d \xi,
$$

which is easily obtained from (5.10) with the aid of (5.5).

(An interesting by-product of the above development, obtained by taking the real parts of (5.6), (5.8), and $(5.9)$, is

$$
\frac{1}{2} \delta_{o p}+\sum_{n=1}^{\infty} J_{2 p}(n x)=\frac{1}{x}+2 \sum_{m=1}^{Q} \frac{\cos \left[2 p \sin ^{-1}(2 m \pi / x)\right]}{\sqrt{x^{2}-(2 m \pi)^{2}}},
$$

where $p=0,1,2, \ldots ; x$ and $Q$ are as specified above. This result was obtained by Ignatowsky [12] by a different method.)

Thus far numerical results for the $\sigma$ 's have been obtained primarily from (5.10). The integrals were evaluated numerically by Paul F. Wacker and William W. Longley, Jr., using Legendre-Gaussian quadrature formulas programed for machine computation. ${ }^{6}$ Values for $\sigma_{p p}^{(2)}$ were calculated from values of $\sigma_{p p}^{(1)}$ using (5.5). Results for the two lowest values of $p$ are given in table 2. (More extensive tables are planned for the previously-mentioned separate publication.)

\section{b. Conversion to More Rapidly Convergent Series}

An alternative method of evaluating the $\sigma$ 's is afforded by a transformation of the Besselfunction series involved into more rapidly convergent series. This was accomplished a good many years ago by W. von Ignatowsky [12] in connection with his work on diffraction by gratings - a subject that will be recognized as being rather closely related to the subject of the present paper. Ignatowsky obtained

$$
\begin{gathered}
\pi \sum_{n=1}^{\infty} Y_{0}(n x)=\log \frac{2 \kappa}{\gamma}+\sum_{n=1}^{Q} \frac{1}{n}+\sum_{n=Q+1}^{\infty}\left(\frac{1}{n}-\frac{\kappa}{\sqrt{n^{2} \kappa^{2}-1}}\right), \\
\pi \sum_{n=1}^{\infty} Y_{2 p}(n x)=\frac{1}{2 p}-\frac{1}{2} \sum_{\mu=1}^{p} \frac{(2 \kappa)^{2 \mu}(p+\mu-1) ! B_{2 \mu}}{(2 \mu) !(p-\mu) !} \\
-\kappa \sum_{n=1}^{Q} \frac{\sin \left[2 p \sin ^{-1}(n \kappa)\right]}{\sqrt{1-n^{2} \kappa^{2}}}-(-)^{p} \kappa \sum_{n=Q+1}^{\infty} \frac{\left(n \kappa-\sqrt{n^{2} \kappa^{2}-1}\right)^{2 p}}{\sqrt{n^{2} \kappa^{2}-1}} .
\end{gathered}
$$

In these expressions $\kappa=2 \pi / x ; p=1,2,3, \ldots$; $Q$ is as defined above; $\log \gamma=0.57721566 \ldots$. . is the Euler-Mascheroni constant; and the $B_{2 \mu}$ are the Bernoulli numbers, here so labeled that $B_{2}=1 / 6, B_{4}=1 / 30, B_{6}=1 / 42, B_{8}=1 / 30, B_{10}=5 / 66$, etc. Equation (5.12a) is fairly well known at the present time. (A few numerical values are given for the series $\sum_{n=1}^{\infty}(-)^{n} Y_{0}(n x)$ in

${ }^{6}$ The estimates of accuracy of computed quantities given in this paper were made by Mr. Longley, who was responsible for most of the detail of the calculations. 
[13].) The series for $\sigma_{11}^{(2)}$ obtained from (5.12a) and (5.12b) has been duplicated by an independent mode of derivation; this represents a partial check of the complicated expression (5.13b). Thus far in the present work, Ignatowsky's series have been used only to spot check some of the numerical results obtained from the definite integrals. The series are especially suitable for either estimation or calculation of the $\sigma$ 's when $p$ is large.

The author has benefited from the discussion of many points with Paul F. Wacker and is indebted to David F. Wait for assistance with some of the theory and computations.

\section{References}

[1] D. S. Saxon, Notes on lectures by Julian Schwinger: Discontinuities in waveguide, Mass. Inst. Tech. Rad. Lab. report $43-7 / 4 / 44$.

[2] F. E. Borgnis and C. H. Papas, Randwertprobleme der Mikrowellenphysik (Springer-Verlag, 1955).

[3] N. Marcuvitz, ed., Mass. Inst. Tech. Rad. Lab. Series 10, 255-257 (McGraw-Hill Book Co., New York, N.Y. 1951).

[4] R. W. Beatty and D. M. Kerns, Recently developed microwave impedance standards and methods of measurement, IRE Trans. on Instrumentation I- $\mathbf{7}, 319$ (1958).

[5] See [2], p. 24.

[6] R. Courant and D. Hilbert, Methods of mathematical physics, 1st Eng. Ed., p. 175 (Interscience, N.Y., 1953).

[7] F. Riesz - B. Sz.-Nagy, Functional analysis, p. 262 (Frederick Ungar Publishing Co., New York, N.Y., 1955).

[8] See [6], p. 474.

[9] P. M. Morse and H. Feshback, Methods of theoretical physics, p. 814 (McGraw-Hill Book Co., Inc., New York, N.Y., 1953).

[10] G. N. Watson, A treatise on the theory of Bessel functions, p. 361 (The University Press, Cambridge, England, 1944).

[11] See [6], p. 468.

[12] W. von Ignatowsky, Über Reihen mit Zylinderfunktionen nach dem Vielfachen des Argumentes, Arch. der Math. u. Phys. 23, 193 (1914).

[13] V. G. Smith, L. Infeld, and W. J. Chien, On some series of Bessel functions, J. Math. and Phys. 20, 22 (1947).

[14] P. Frank and R. v. Mises, Die Differential- und Integralgleichungen der Mechanik und Physik, Vol. 2, p. 808ff (Mary Rosenberg, New York, N.Y., 1943).

Boulder, Colo.

(Paper 64B2-29) 


\section{Publications of the National Bureau of Standards*}

\section{(Including papers in outside journals)}

\section{Selected Abstracts}

A comparison of experimental and theoretical relations between Young's modulus and the flexural and longitudinal resonance frequencies of uniform bars, S. Spinner, T. W. Reichard, and W. E. Tefft, J. Research NBS 64A, No. 2, $147^{7}$ (1960).

The relations between Young's modulus and the mechanical resonance flexural and longitudinal frequencies of two sets of steel bars has been established by an empirical method. The agreement between theoretical and experimental curves for flexural vibrations appeared somewhat better than that for longitudinal vibrations.

On the mode theory of very-low-frequency propagation in the presence of a transverse magnetic field, D. D. Crombie, J. Research NBS 64D, No. 3, 265 (1960).

The effect of a purely transverse horizontal magnetic field on the propagation of VLF waves is considered. It is shown that the magnetic field introduces non-reciprocity, and that for propagation along the magnetic equator, the rate of attenuation is less for west-to-east propagation than for eastto-west propagation.

On the theory of reflection of low- and very-lowradiofrequency waves from the ionosphere, $\mathrm{J}$. R. Johler and L. C. Walters, J. Research NBS 64D, No. 3, 269 (1960).

The rigorous application of the magneto-ionic theory to the calculation of reflection coefficients for a sharply bounded ionosphere model is discussed. This is a comprehensive paper on the details which pertain to the rigorous evaluation of the reflection coefficients. The paper is illustrated with computations applicable to the $D$-region or the $E$-region of the ionosphere. The quasi-longitudinal approximation is derived from this theory and the range of validity of this approximation is illustrated. The restrictions imposed by the use of a sharply bounded model ionosphere are discussed.

Focusing, defocusing, and refraction in a circularly stratified atmosphere, K. Toman, J. Research NBS 64D, No. 3, 289 (1960).

Focusing, defocusing, astronomical refraction and path length of rays as a function of the departure angle $\Delta$ of the ray at the source is described for cases with the source outside, inside, or on the boundary of a circular stratification. Relative to zero elevation angle symmetrical and centrosymmetrical distributions are found.

Impedance characteristics of a uniform current loop having a spherical core, S. Adachi, J. Research NBS 64D, No. 3, 297 (1960).

The radiation impedance is derived by the EMF method in a convenient form as the sum of the self-radiation impedance of a loop in the free space and an additional term due to the reaction between the loop and the sphere which is proportional to the well-known expansion coefficient of a magnetictype scattered wave from a sphere in an incident plane wave. The first antiresonance frequency has been given in the form of a universal curve for a very small uniform current loop with core of an arbitrary composition of $\mu_{s}$ and $\epsilon_{s}$, subject to the condition that the refraction coefficient $N=\sqrt{\mu_{s} \epsilon_{s}}$ is extremely large. Some numerical calculations show that high- $\mu$ core is desirable for a comparatively lower frequency region, and high- $\epsilon$ core is rather desirable in an antiresonance region.

Basic theorems in matrix theory, M. Marcus, NBS Applied Math. Series 57 (1960) 15 cents.

This is a survey of the basic identities and inequalities of matrix theory. Included are results dealing with elementary properties, canonical forms, invariance, congruence, commutativity, orthogonalization, eigenvalues, determinants, submatrices, rank, determinant and rank inequalities, numerical methods for inversion and eigenvalues, condition numbers.

Relaxation processes in multistate systems, K. E. Shuler, Phys. of Fluids $\boldsymbol{2}$, No. 4, 442 (1959).

An analysis is made of the relaxation of "multistate" systems, i.e., systems with more than two quantum states or two different chemical species, for linearized processes described by the "Master Equation" of the theory of transport processes. These results are compared with those obtained from the analysis of two-state relaxation processes and the concept of "relaxation time" is discussed in this framework. A discussion is presented of the transformation of microscopic to macroscopic relaxation equations. The existence of periodic and/or aperiodic oscillatory solution of the linear multistate relaxation equation is investigated. It is shown that the multistate relaxation equations admit of aperiodic oscillatory solutions.

Invariant and complete stress functions for general continua, C. Truesdell, Arch. Rational Mech. Anal. 1, No. 4, 1 (1959).

This paper presents an organized survey of results, with proofs, concerning exact general solution of the underdetermined equations of motion of a continuous medium. Special attention is given to a flat space of $n$ dimensions, to an $n$-dimensional space of constant curvature, and to a 2-dimensional surface. Two methods are used: (1) the representation of a solenoidal vector as the curl of a vector potential, and (2) a variational formulation, essentially the converse of the principle of virtual work.

The construction of Hadamard matrices, E. C. Dade and K. Goldberg, Mich. Math. J. 6, 247 (1959).

It is proved that a Hadamard matrix of order $4 n$ can be constructed if there exists a transitive permutation group of odd order and degree $4 n-1$ whose subgroups leaving one element fixed have three transitivity sets each.

\section{Bending and stretching of corrugated diaphragms,} R. F. Dressler, ASME Trans. 81D, No. 4, 651 (1959).

Solutions of the exact linear elastic shell equations for all stresses and displacements in a typical corrugated diaphragm are presented for three cases over a 9 to 1 range of thicknesses. Results were obtained by numerical integration in an electronic digital computer. The effect of thickness variation is discussed with respect to both stresses and resultants, and peak values needed for design purposes are presented. Circumferential and meridional stresses are found to be equally important throughout the thickness range analyzed. Bending and membrane stresses are likewise equally important throughout the range. Peak values in some cases occur near the outer rim. 
Modular forms whose coefficients possess multiplicative properties, M. Newman, Ann. Math. \%o, $478(1959)$.

Let $q$ be a prime, $r$ and $s$ nonzero integers, and $\eta(\tau)$ the Dodekind modular form. All modular forms of type $\eta^{r}(\tau) \eta^{s}(q \tau)$ such that the related Dirichlet series possesses an Euler product are determined.

The analysis of latin squares with a certain type of row-column interaction, J. Mandel, Technometrics 1, No. 4, 379 (1959).

A serious limitation in the use of latin squares is the confounding of the main effect of each factor with the interaction of the remaining two factors. In some cases, the interaction of rows and columns can be expressed as a multiplicative term of assigned factors associated with rows and columns. The analysis of such designs is presented in detail. An example is discussed. The method is extended to the case where the treatment effects are linearly related to a given concomitant variable. A brief discussion is given of the relation of the proposed method with other tests for nonadditivity.

A note on algebras, A. J. Goldman, Am. Math. Mo. 66, 795 (1959).

The usual sufficient conditions that both regular representations of an algebra $A$ be faithful are shown not to be necessary. A necessary and sufficient condition (viz, that no nonzero member of $A$ be either a left annihilator or a right annihilator) is derived.

Linear differential equations of the second order with a large parameter, F. W. J. Olver, J. Soc. Indust. Appl. Math. \%, 306 (1959).

An investigation is made of the differential equation

$$
\frac{d^{2} w}{d z^{2}}=\left\{u^{2} p(Z)+q(Z)\right\} w
$$

for large values of the parameter $u$.

\section{List of Titles}

Journal of Research, Section 64A, No. 2, MarchApril 1960.70 cents.

A carbon-14 beta-ray standard, benzoic acid-7-C14 in toluene, for liquid scintillation counters. W. F. Marlow and R. W. Medlock.

A comparison of experimental and theoretical relations between Young's modulus and the flexural and longitudinal resonance frequencies of uniform bars. S. Spinner, T. W. Reichard, and W. E. Tefft. (See above abstracts.)

Determination of copolymer composition by combustion analysis for carbon and hydrogen. Lawrence A. Wood, Irving Madorsky, and Rolf A. Paulson.

Some effects of aging on the surface area of portland cement paste. C. M. Hunt, L. A. Tomes, and R. L. Blaine.

Conformations of the pyranoid sugars. I. Classification of conformers. H. S. Isbell and R. S. Tipson.

Tritium-labeled compounds III. Aldoses $-1-t$. H. S. Isbell, H. L. Frush, N. B. Holt, and J. D. Moyer.

Determination of aluminum in precipitation hardening stainless steel and high temperature alloys. Lawrence A. Machlan, John L. Hague, and Edward J. Meros.

\section{Journal of Research, Section 64D, No. 3, May-June} 1960. 70 cents.

A theory of radar scattering by the moon. T. B. A. Senior and K. M. Siegel.

A theory of wavelength dependence in ultrahigh frequency transhorizon propagation based on meteorological considerations. Ralph Bolgiano, Jr.
A preliminary study of radiometeorological effects on beyondhorizon propagation. F. Ikegami.

Trade-wind inversion as a transoceanic duct. M. Katzin, H. Pezzner, B. Y.-C. Koo, J. V. Larson, and J. C. Katzin. An analysis of propagation measurements made at 418 megacycles per second well beyond the radio horizon (a digest). H. B. Janes, J. C. Stroud, and M. T. Decker.

On the calculation of the departures of radio wave bending from normal. B. R. Bean and E. J. Dutton.

On the mode theory of very-low-frequency propagation in the presence of a transverse magnetic field. D. D. Crombie. (See above abstracts.)

On the theory of reflection of low- and very-low-radiofrequency waves from the ionosphere. J. Ralph Johler and Lillie C. Walters. (See above abstracts.)

Focusing, defocusing, and refraction in a circularly stratified atmosphere. K. Toman. (See above abstracts.)

Response of a loaded electric dipole in an imperfectly conducting cylinder of finite length. Charles W. Harrison, Jr., and Ronald W. P. King.

Impedance characteristics of a uniform current loop having a spherical core. Saburo Adachi. (See above abstracts.)

\section{Journal of Research, Section 64C, No. 2, April-June} 1960. 75 cents.

Measurement of cobalt-60 and cesium-137 gamma rays with a free-air chamber. H. O. Wyckoff.

Apparatus for the measurement of the normal spectral emissivity in the infrared. Arthur G. Maki, Ralph Stair, and Russell G. Johnston.

Electrostatic deflection plates for cathode-ray tubes. I. Design of single-bend deflection plates with parallel entrance sections. II. Deflection defocusing distortion of single-bend deflection plates with parallel entrance sections. Lothar Frenkel.

The functional synthesis of linear plots. J. P. Vinti and R. F. Dressler.

Radiation field from a rectangular source. J. H. Hubbell, R. L. Bach, and J. C. Lamkin.

Microwave attenuation measurements with accuracies from 0.0001 to 0.06 decibel over a range of 0.01 to 50 decibels. G. F. Engen and R. W. Beatty.

Effect of oleophobic films on metal fatigue. H. E. Frankel, J. A. Bennett, and W. L. Holshouser.

Ratio-recording spectroradiometer. Harry K. Hammond III, Warren L. Holford, and Milton L. Kuder.

An intermittent-action camera with absolute time calibration. G. Hefley, R. H. Doherty, and E. L. Berger.

Screw-thread standards for federal services 1957, Amends in part H28 (1944) (and in part its 1950 Supplement), NBS Handb. H28 (1957) - Pt. II (1959) 75 cents.

Specification for dry cells and batteries (supersedes Circular 559), NBS Handb. 71, (1959) 25 cents.

Report of the 44th National Conference on Weights and Measures 1959, NBS Misc. Publ. 228, (1959) 65 cents.

Research Highlights of the National Bureau of Standards Annual Report, Fiscal Year 1959, NBS Misc. Publ. 229 (1959) 55 cents.

Distribution of mail by destination at the San Francisco, Los Angeles, and Baltimore Post Offices, N. C. Severo and A. E. Newman, NBS Tech. Note 27 (PB151386) (1959) $\$ 1.50$.

A history of vertical-incidence ionosphere sounding at the National Bureau of Standards, S. C. Gladden, NBS Tech. Note 28 (PB151387) (1959) $\$ 2.00$

Photographic dosimetry at total exposure levels below $20 \mathrm{mr}$, M. Ehrlich and W. L. McLaughlin, NBS Tech. Note 29 (PB151388) (1959) 50 cents.

An atlas of oblique-incidence ionograms, V. Agy, K. Davíes, and R. Salaman, NBS Tech. Note 31 (PB151390) (1959) $\$ 2.25$.

Resistance diode bridge circuit for temperature control, L. H. Bennett and V. M. Johnson, NBS Tech. Note 34 (PB151393) (1959) 50 cents. 
Service area of an airborne television station, M. T. Decker, NBS Tech. Note 35 (PB151394) (1959) 75 cents.

Rotational transfer in the fluorescence spectrum of $\mathrm{OH}\left({ }^{2} \Sigma^{+}\right)$, T. Carrington, J. Chem. Phys. 31, No. 5, 1418 (1959).

Determination of viscosity, J. F. Swindells, Ch. XII, Physical methods of organic chemistry, pt. I, p. 689 (Interscience Publishers Inc., New York, N.Y., 1959).

Triaxial tension at the head of a rapidly running crack in a plate, J. M. Frankland, Trans. ASME [E] 26, No. 4, 570 (1959)

Poly(tetrafluorethylene) - a radiation-resistant polymer, L. A Wall and R. E. Florin, Letter to Ed., J. App. Polymer Sei. II, No. 5, 251 (1959).

On the approximate daytime constancy of the absorption of radio waves in the lower ionosphere, $\mathrm{S}$. Chapman and $\mathrm{K}$. Davies, J. Atmospheric and Terrest. Phys. 13, No. 1-2, 86 (1958).

An X-ray crystallographic study of Schroeckingerite and its dehydration product, D. K. Smith, Am. Mineralogist 44, 1020 (1959)

A note on the scattering of electrons from atomic hydrogen, A. Temkin, Phys. Rev. 116, No. 2, 358 (1959).

Design of free-air ionization chambers for the soft X-ray region $(20-100 \mathrm{kv})$, V. H. Ritz, Radiology $\mathbf{7 3 ,}$, No. 6, 911 (1959).

Thermal conductivity of indium antimonide at low temperatures, E. V. Mielczarek and H. P. R. Frederikse, Phys. Rev. 115, 888 (1959).

Model for vibrational relaxation of diatomic gases behind shock waves, R. Herman and R. J. Rubin, Phys. of Fluids 2, No. 5, 547 (1959).

Absolute photometry of the aurora-I. The ionized molecular nitrogen emission and the oxygen green line in the dark atmosphere, M. H. Rees, J. Atmospheric and Terrest. Phys. 14, 325 (1959).
Direction findings on whistlers, J. M. Watts, J. Geophys. Research 64, No. 11, 2029 (1959).

Geomagnetic and ionospheric phenomena associated with nuclear explosions, S. Matsushita, Nature 184, BA33 (1959).

Stress corrosion cracking in low carbon steel, H. L. Logan, Proc. Phys. Met. Stress Corrosion Fracture Conf. Pittsburgh, Pa., Apr. 2-3, 1959, reprinted from Met. Soc. Conf., p. 295 (Interscience Publishers, New York, N.Y., 1959).

Higher oxides of silver, H.E. Swanson, J. Electrochem. Soc. 106, No. 12, 1073 (1959).

Oscillatory behavior of magnetic susceptibility and electronic conductivity, A. H. Kahn and H. P. R. Frederikse, vol. IX, Advances in Solid State Physics, p. 257 (Academic Press Inc., New York, N.Y., 1959).

Geomagnetic activity following large solar flares, C. S. Warwick and R. T. Hansen, J. Atmospheric and Terrest. Phys. 14, 287 (1959).

Simplification of systems of units currently evolving in the electrical field, F. B. Silsbee, Systems of Units, Am. Assoc. Advance. Sci., p. 7 (1959).

Atoms and free radicals by $\gamma$-irradiation at $4.2^{\circ} \mathrm{K}, \mathrm{L}$. A. Wall, D. W. Brown, and R. E. Florin, J. Phys. Chem. 63, 1762 (1959).

*Publications for which a price is indicated (except for NBS Technical Notes) are available only from the Superintentent of Documents, U.S. Government Printing Office, Washington 25, D.C. (foreign postage, one-fourth additional). Technical Notes are available only from the Office of Technical Services, U.S. Department of Commerce, Washington 25, D.C. (Order by PB number). Reprints from outside journals and the NBS Journal of Research may often be obtained directly from the authors. 